\title{
Luminosity Function of Gamma-Ray Bursts Derived Without Benefit of Redshifts
}

\author{
Maarten Schmidt \\ California Institute of Technology, Pasadena, CA 91125 \\ mxs@deimos.caltech.edu
}

\begin{abstract}
We show that the Euclidean value of $\left\langle V / V_{\max }>\right.$ for gamma-ray bursts (GRB) selected on a timescale of $1024 \mathrm{~ms}$ is correlated with spectral hardness. The value of $<V / V_{\text {max }}>$ ranges from $\sim 0.42$ for soft bursts to $\sim 0.26$ for the hardest bursts. Given that the Euclidean value of $<V / V_{\max }>$ for cosmological objects in a well defined sample is a distance indicator, the hard bursts must reside at larger redshifts and therefore be more luminous than the soft bursts. The resulting luminosity-hardness correlation cannot be shown explicitly due to the small number of observed GRB redshifts at the present time. Based on the $<V / V_{\max }>$-hardness correlation, we derive the luminosity function of GRBs without using any redshifts, but we have to make an assumption how the comoving GRB rate varies with redshift. We present luminosity functions for three models of the GRB rate as a function of redshift, based on star formation rates. The peak luminosity functions are approximately broken power laws with an isotropic-equivalent break luminosity of $\sim 10^{51.5} \mathrm{erg} \mathrm{s}^{-1}$ in the $50-300 \mathrm{keV}$ range and total local rate densities of $\sim 0.5 \mathrm{Gpc}^{-3} \mathrm{y}^{-1}$. Predicted GRB counts as a function of flux and redshift are presented. Based on the GRB luminosity function, we carry out a simulation to produce the luminosity-hardness correlation, which shows that the hardest GRBs are $\sim 20$ times more luminous than the softest ones.
\end{abstract}

Subject headings: cosmology: observations — gamma rays: bursts

\section{Introduction}

Since there are only a small number of gamma-ray bursts (GRBs) with observed redshifts at the present time, the derivation of their luminosity function has presented a difficult problem. A well established GRB luminosity function would allow predictions of the distributions of flux and of redshift for planned GRB surveys. The space densities and luminosities of GRBs would be useful in discussing their relation to other objects and in understanding the physics of these fascinating objects. Usually, the luminosity function of extragalactic objects is derived from a set of samples of the objects with observed redshifts to well defined flux limits. Within a given cosmological 
model, such observations allow a derivation of the luminosity function, including its dependence on redshift.

The Burst and Transient Source Experiment (BATSE) (Fishman et al. 1989) on board the Compton Gamma-Ray Observatory has made possible the collection of large well defined GRB samples, such as the BATSE catalog ${ }^{1}$ based on on-board triggers, and the BD2 sample (Schmidt 1999a,b) based on BATSE DISCLA data (see Sec. 2). Redshifts are available for a relatively small number of GRBs. At the present time no well defined sample of GRBs can be formed in which all or a substantial fraction of the objects has an observed redshift. As a consequence various other methods have been used to derive the GRB luminosity function.

The observed $\log N-\log P$ relation in a survey carries information about the luminosity of the survey objects. For a given cosmological model, the flux at which the $\log N-\log P \operatorname{relation}$ starts to fall below the Euclidean slope of $-3 / 2$ allows derivation of the corresponding luminosity. Generally, the analyses assumed that GRBs were standard candles (Cohen \& Piran 1995; Pendleton et al. 1996; Wijers et al. 1998; Totani 1999) and derived the maximum redshift or the standard candle luminosity by fitting the observed $\log N-\log P$ distribution for a given cosmological model and an assumed GRB density distribution.

A different method to derive the luminosity function makes use of a luminosity indicator, i.e., an observable property that correlates with luminosity. Several luminosity indicators have been proposed, such as the spectral lag derived from cross-correlation of two spectral channels (Norris, Marani \& Bonnell 2000), and the variability in the time profile (Fenimore \& Ramirez-Ruiz 2000). The correlations of these properties with luminosity have been discovered and calibrated from a relatively small number of GRBs with redshifts, e.g., the variability correlation is based on seven redshifts (Fenimore \& Ramirez-Ruiz 2000).

We have previously explored a reverse approach, in which we made assumptions about the shape and extent of the luminosity function, and then used the Euclidean $\left\langle V / V_{\max }\right\rangle$ value of the BD2 sample (see Sec. 2) to derive the characteristic luminosity $L^{*}$ and the local GRB space density, again for a given cosmological model and GRB space distribution (Schmidt 1999b). Varying the assumptions about the shape of the luminosity function allowed an evaluation of the sensitivity of $L^{*}$ and the space density to the various input parameters.

The deviation of the Euclidean $\left\langle V / V_{\max }>\right.$ of cosmological objects in a well defined sample from the value 0.5 reflects to first order the effect of using Euclidean geometry in its derivation rather than an appropriate relativistic cosmological model. These effects increase with distance and therefore the Euclidean $<V / V_{\max }>$ is a distance indicator for the sample objects. We illustrate this in Figure 1, where we plot the maximum redshift of a sample of bursts versus the Euclidean $<V / V_{\max }>$ for standard candle GRBs with a photon spectrum $\sim E^{-2}$. The curves show the

\footnotetext{
${ }^{1}$ The BATSE catalog (C. A. Meegan et al. 1999) is available at http://gammaray.msfc.nasa.gov/batse/grb/ catalog/current/.
} 
relation for two different scenarios for the comoving space distribution. Clearly, the Euclidean value of $<V / V_{\max }>$ is a cosmological distance indicator that can be used to determine a distance scale for cosmological objects for which no redshifts are available.

In this paper, we will demonstrate a correlation between the Euclidean value of $\left\langle V / V_{\max }\right\rangle$ and the spectral hardness of GRBs. We interpret this correlation in terms of a luminosity-hardness correlation but are initally unable to show the correlation explicitly. We use the $\left\langle V / V_{\max }>\right.$ hardness correlation to derive the GRB luminosity function, as well as predicted counts as a function of flux and redshift. Finally, we show the results of a simulation producing the luminosity-hardness correlation.

The paper is organized as follows. In Sec. 2, we present the observed correlation between spectral hardness and $\left\langle V / V_{\max }\right\rangle$. The methodology used to derive the luminosity function is discussed in Sec. 3. The resulting luminosity functions are shown in Sec. 4, together with predicted distributions of flux and redshift. We show the results of a simulation producing the luminosityhardness correlation in Sec. 5, followed by the discussion in Sec. 6. Throughout this paper, we will be using a flat cosmological model with $H_{0}=65 \mathrm{~km} \mathrm{~s}^{-1} \mathrm{Mpc}^{-1}, \Omega_{M}=0.3$, and $\Omega_{\Lambda}=0.7$ (Bahcall et al. 1999).

\section{Spectral Hardness as a Luminosity Indicator}

\subsection{Data}

In this paper we use a large homogeneous sample, the BD2 sample, derived from BATSE DISCLA data consisting of the continuous data stream from the eight BATSE LAD detectors in four energy channels on a timescale of $1024 \mathrm{~ms}$ (Fishman et al. 1989). The sample was derived using a software trigger algorithm that interpolated the background between given times before and after the onset of the burst and required an excess of at least $5 \sigma$ over background in at least two detectors in the energy range $50-300 \mathrm{keV}$ (Schmidt 1999a). The first version (the BD1 sample) was described in Schmidt (1999a). A revision discussed in Sec. 2 of Schmidt (1999b) produced the BD2 sample.

The BD2 sample covers a period of 5.9 y from TJD 8365 - 10528. It contains 1391 GRBs, of which 1013 are also listed in the BATSE catalog. The median photon flux limit of the BD2 sample over the energy range $50-300 \mathrm{keV}$ is $0.31 \mathrm{ph} \mathrm{cm}^{-2} \mathrm{~s}^{-1}$. The average Euclidean $V / V_{\max }$ is $0.336 \pm 0.008$. The sample of 1391 GRBs effectively represents $2.003 \mathrm{y}$ of full sky coverage.

In studying the correlation of spectral hardness of GRBs with other properties, we have to choose a relevant part of the light curve since the spectral hardness of GRBs generally varies while the burst is going on. The Euclidean values of $V / V_{\max }$ in the BD2 sample have been derived from simulations in which the GRB is moved out until the detection algorithm fails to trigger (Schmidt 1999a). In most cases, the final detection is on the peak of the GRB time profile. Therefore, we 
use the $1024 \mathrm{~ms}$ interval containing the peak flux to derive the hardness ratio HR32 as the ratio of the burst counts in BATSE channels $3(100-300 \mathrm{keV})$ and $2(50-100 \mathrm{keV})$ for the brightest illuminated detector. From HR32, the illumination angle and the BATSE detector response matrix, we then derive the photon spectrum slope $\alpha_{23}$.

In Figure 2, we plot the Euclidean value of $V / V_{\max }$ versus $\alpha_{23}$ for all 1391 GRBs in the BD2 sample. We also show $<V / V_{\max }>$ for 4 spectral classes of $\sim 348$ GRBs each (see Table 1 ). The $<V / V_{\max }>$ values range from $\sim 0.47$ for the softest bursts to $\sim 0.27$ for the hard ones. The mean errors of the $<V / V_{\max }>$ values are around \pm 0.016 .

Before we use this correlation between $\left\langle V / V_{\max }>\right.$ and $\left\langle\alpha_{23}\right\rangle$, we evaluate the effect of statistical errors in the counts used to derive $\alpha_{23}$. The detection of GRBs in the BD2 sample was based on the sum of the counts in channels 2 and 3, without regard to their ratio HR32. The statistical error in HR32, determined by the burst counts and the background counts in each of the two channels, will be larger for weaker GRBs, which have larger values of $V / V_{\max }$. We see in Figure 2 a strong concentration of $\alpha_{23}$ around -1.5 at low $V / V_{\max }$ where errors are small. As we move upward in Figure 2, the statistical errors will scatter $\alpha_{23}$ horizontally, causing the concentration at $\alpha_{23} \sim-1.5$ to widen. There will be a net movement of points to the left and to the right and this effect will increase toward the top of the diagram. This causes $\left\langle V / V_{\max }\right\rangle$ to be too large for $\alpha_{23}$ values well below and above -1.5 .

We have carried out simulations to estimate the systematic effect on $<V / V_{\max }>$. We used actually measured background counts in channels 2 and 3 for each of the 1391 GRBs and took the errors to be gaussian. We assumed that the actual distribution of $\alpha_{23}$ is represented by the brightest quartile of $348 \mathrm{GRBs}$, which are confined to $V / V_{\max }<0.069$ at the very bottom of Figure 2. We ensured that in the input sample there was no correlation between $\left\langle V / V_{\max }\right\rangle$ and $\alpha_{23}$ by associating each $V / V_{\max }$ with each $\alpha_{23}$, so that the input set of bright bursts consisted of $348 \times 348$ objects.

This hypothetical bright set was then used to simulate $V / V_{\max }$ and $\alpha_{23}$ values for the 1043 objects with $V / V_{\max }>0.069$. The results are exhibited in Table 1 and Figure 2, where we show both observed values of $\left\langle\alpha_{23}\right\rangle$ and $\left\langle V / V_{\max }\right\rangle$, and values corrected for the systematic errors resulting from the simulations. For the softest and the hardest classes, the errors caused $\alpha_{23}$ to move away from the center and $<V / V_{\max }>$ to increase. For the classes in the middle, $<V / V_{\max }>$ decreased because high values of $V / V_{\max }$ scattered away in $\alpha_{23}$. We are not showing mean errors for the corrected values of $\left\langle V / V_{\max }\right\rangle$. They will be at least \pm 0.016 but are likely dominated by systematic errors in the simulations or in the statistical behavior of the observed counts that we cannot judge. 


\subsection{Interpretation as Luminosity-Hardness Correlation}

Given that the Euclidean value of $\left\langle V / V_{\max }\right\rangle$ is a distance indicator (see Fig. 1), the hard bursts in the sample on the average must be at larger distances and the softer bursts nearer. With the density distribution SF2 (see Sec. 3) and the assumption of standard candles used for the illustration in Figure 1, one would estimate a maximum redshift of $\sim 1.4$ for the softest bursts and $\sim 3.4$ for the hardest bursts. With the given flux limit of the BD2 sample, the soft bursts are therefore of lower luminosity and the hard bursts of higher luminosity. We conclude that the spectral index $\alpha_{23}$ is a luminosity indicator and that there exists a luminosity-hardness correlation.

At this stage we cannot show the luminosity-hardness correlation explicitly due to the dearth of observed redshifts. Once we have derived the GRB luminosity function, we will produce an explicit luminosity-hardness correlation through simulation, see Sec. 5.

Could the correlation of $<V / V_{\max }>$ on $\alpha_{23}$ be a consequence of the shape of the spectrum of GRBs? The typical GRB photon spectrum is often characterized as the Band spectrum (Band et al. 1993), with low energy slope $\sim-1$, high energy slope $\sim-2$ and break energy near $150 \mathrm{keV}$. At low redshift, this spectrum will have an observed $\alpha_{23}$ between -1 and -2 , while at high redshift it will be -2 . Thus more distant bursts will have softer spectra, which is the opposite of our finding above.

Previous studies of the correlation between hardness and global GRB properties have been based on the $\log N-\log P$ relation or on $V / V_{\max }$, often involving duration-hardness classes (Belli 1992, 1996; Kouveliotou, et al. 1993, 1996; Pendleton et al. 1998; Tavani 1998). Among the three classes considered by Tavani (1998), classes B and C contained GRBs with $T_{90}>2.5 \mathrm{~s}$ similar to the BD2 GRBs detected at a time scale of $1024 \mathrm{~ms}$. The hard bursts in class B had $<V / V_{\max }>$ $=0.29$ while the soft bursts in class $\mathrm{C}$ had $<V / V_{\max }>=0.42$, showing the same trend as seen in Figure 2. Also of interest is the study by Pendleton et al. (1998) who defined NHE bursts as those that have a marked lack of high-energy flux $(E>300 \mathrm{keV})$, in contrast to HE bursts that have a strong high-energy flux. Using the $\log N-\log P$ relations for both types they concluded that HE bursts are eight times more luminous than NHE bursts. This agrees qualitatively with our finding that hard bursts are more luminous than soft bursts.

We have not used the durations or the duration-hardness classification of GRBs for the following reason. In the BD2 sample we define the duration of a GRB as the total time elapsed between the time of trigger and the last time the burst flux exceeded the limiting flux for triggering. In our simulations in which we move a burst out in distance to derive its $V / V_{\max }$, we find that the duration decreases to around 1 or 2 seconds when last detected. Clearly, our definition of 'duration' does not produce an absolute property of the burst. Therefore, we have not considered duration-hardness classes for the BD2 GRBs. The BATSE $T_{90}$ and $T_{50}$ durations also suffer from a "fluence-duration" bias according to Hakkila et al. (2000). If the derived duration of a given burst depends on its flux, then the $V / V_{\max }$ values for bursts with a minimum observed duration have to be derived using two simultaneous limits (Schmidt 1968), i.e. the flux limit and the duration limit. Ignoring this 
requirement will give rise to systematic errors in $V / V_{\max }$.

\section{Deriving the GRB Luminosity Function From $<V / V_{\max }>$}

In the next section, we will find that the central luminosities of the 4 spectral classes (see Table 1) range over a factor of around $10-50$. This makes it possible to construct the luminosity function of GRBs. The derivation for each of the spectral classes is similar to that employed previously in Schmidt (1999b) for the entire luminosity function. We use the cosmological parameters $H_{0}=$ $65 \mathrm{~km} \mathrm{~s}^{-1} \mathrm{Mpc}^{-1}, \Omega_{M}=0.3$, and $\Omega_{\Lambda}=0.7$.

We assume that the GRB luminosity function $\Phi(L, z, s p)$ of the spectral class $s p$ can be written as

$$
\Phi(L, z, s p)=\Phi_{0}(L, s p) R_{\mathrm{GRB}}(z),
$$

where $s p$ refers to the four spectral classes (see Table 1), $L$ is the peak luminosity in the given energy band, $\Phi_{0}(L, s p)$ is the $z=0$ luminosity function of class $s p$, and $R_{\mathrm{GRB}}(z)$ the comoving GRB density distribution normalized at $z=0$. We assume that each $\Phi_{0}(L, s p)$ has a gaussian distribution of $\log L$ with a dispersion $\sigma_{\log L}$ around a central peak luminosity $L_{c}$. The GRB luminosity function $\Phi_{0}(L)$ is the sum of the spectral luminosity functions $\Phi_{0}(L, s p)$.

We assume that the photon spectrum is proportional to $E^{\alpha_{23}}$. The peak flux $P(L, z)$ observed for a GRB of luminosity $L$ at redshift $z$ is

$$
P(L, z)=\frac{L}{4 \pi A^{2}(z)}(1+z)^{\left(2+\alpha_{23}\right)},
$$

where $A(z)$ is the bolometric luminosity distance for the cosmological model. The peak flux $\log N-$ $\log P$ distribution for GRBs of spectral class $s p$ is,

$$
N(>P, s p)=\int \Phi_{o}(L, s p) d \log L \int_{0}^{z(L, P, s p)} R_{G R B}(z)(1+z)^{-1}(d V(z) / d z) d z
$$

where $z(L, P, s p)$ is derived from equation $(2), V(z)$ is the comoving volume and the term $(1+z)^{-1}$ represents the time dilation (Totani 1999). With the known distribution of flux limits $P_{\text {lim }}$ in the BD2 sample, we can derive the Euclidean value of $<V / V_{\max }>$ from the individual values $V / V_{\max }$ $=\left(P / P_{\text {lim }}\right)^{-3 / 2}$. The central peak luminosity $L_{c}$ of each spectral class is iterated until $\left\langle V / V_{\max }>\right.$ agrees with the observed value.

The limiting peak flux $P_{\text {lim }}$ depends on the GRB spectrum. Based on the BATSE detector response matrix, $\log P_{\text {lim }}$ increases by $\sim 0.11$ from the softest to the hardest class. This does not

affect the $V / V_{\max }$ derivation described above since $P$ is equally affected. The luminosity $L_{c}$ derived for spectral class $s p$ scales as $P_{\text {lim }}$.

The comoving GRB density distribution $R_{\mathrm{GRB}}(z)$ is often referred to as the 'star formation rate' based on the expectation that GRBs are caused by massive stars. Porciani \& Madau (2000) 
have parametrized various models for the evolution of the cosmic star formation rate (SFR) with redshift. In model SF1, based on Madau \& Pozetti (2000), the SFR rises rapidly by an order of magnitude between $z=0$ and $z=1$, peaks between $z=1$ and $z=2$ and declines gently at higher redshifts. In model SF2, based on Steidel et al. (1999), it rises similarly but then remains roughly constant for $z>2$. Model SF3, reflecting the possibility that extinction has been underestimated (Blaine et al. 1999), has an SFR continuing to rise beyond $z=2$. For the cosmological model used in this paper $\left(H_{0}=65 \mathrm{~km} \mathrm{~s}^{-1} \mathrm{Mpc}^{-1}, \Omega_{M}=0.3\right.$, and $\left.\Omega_{\Lambda}=0.7\right)$, the star formation rates for $z=1,3,5$ are $9.5,7.7,3.4$ (SF1), 8.3, 12.8, 12.7 (SF2), and 6.2, 12.8, 16.1 (SF3), respectively. We use these three models to characterize $R_{\mathrm{GRB}}(z)$ in the derivation of the luminosity function.

\section{The GRB Luminosity Function}

As descibed in the preceding section, we derive the luminosity function at $z=0$ for each of the 4 spectral classes separately. We set the gaussian dispersion of each $\Phi_{0}(L, s p)$ at $\sigma_{\log L}=0.4$. The central peak luminosities $L_{c}$ of the spectral classes were determined from the corrected values of $\left\langle\alpha_{23}>\right.$ and $\left\langle V / V_{\max }>\right.$ given in Table 1 . The resulting luminosity functions at $z=0$ for each of the four spectral classes for the SF2 model are shown in Figure 3.

The sum of the spectral luminosity functions constitutes the overall luminosity function. Figure 4 shows the resulting luminosity functions for density distributions SF1, SF2, and SF3. The central peak luminosities $\log L_{c}$ range from $50.32-51.29,50.27-51.57$, and $50.18-51.88$, re-

spectively. The luminosity function generally appears to be a power law from $\sim 10^{50.5} \mathrm{erg} \mathrm{s}^{-1}$ to $\sim 10^{51.5} \mathrm{erg} \mathrm{s}^{-1}$, and then to decline more steeply. The total $z=0$ GRB densities are 0.48, 0.51, and $0.72 \mathrm{Gpc}^{-3} \mathrm{y}^{-1}$ for SF1, SF2, and SF3, respectively. The luminosities and densities quoted are 'isotropic-equivalent' values. If all GRBs are beamed into, say, $\omega$ steradians, then luminosities require multiplication by $\omega / 4 \pi$ and densities by $4 \pi / \omega$. If the luminosity-hardness correlation represents the distribution of luminosities within a GRB beam, the situation would be more complex and the corrections to luminosity and density would be a function of luminosity.

The cumulative distribution of peak fluxes observed in the BD2 sample is shown in Figure 5. The predicted $\log N-\log P$ distributions are in excellent to good agreement with the observations. Compared to an annual all-sky rate of 694 GRBs based on the BD2 sample, we expect above 0.1 (0.01) $\mathrm{ph} \mathrm{cm}^{-2} \mathrm{~s}^{-1}$ annual rates of 2560 (5090), 2720 (6810), and 2830 (8460) for cases SF1, SF2, and SF3, respectively.

In Figure 6, we show histograms of the expected redshift distribution in the BD2 sample. The fraction of high redshifts increases from SF1 to SF2 to SF3: the expected fractions with $z>4$ are 1,5 , and $12 \%$, respectively. The largest single redshift that may be expected in the BD2 sample of 1391 GRBs on the basis of these three models is around 6, 13, or 19, respectively. These, however, may be overestimates for SF2 and SF3, since in these cases the star formation rate remains high at large redshift, with no provision for the onset of star formation. 
In the derivation of the luminosity function, we assumed that the spectrum of the GRBs in each spectral classes was a simple power law of slope $\alpha_{23}$. Belli (2000) has compiled GRB spectra as a function of a fluence hardness ratio, based on the four BATSE LAD channels. Most of the spectra appear to be broken power laws. The dependence on the hardness ratio seems to be a general change in slopes with little change in break energy. We have tested the effect of using these systematics by adopting instead of the simple power law a Band spectrum (Band et al. 1993) with $\alpha=\alpha_{23}+1.0, \beta=\alpha_{23}$ and $E_{0}=150 \mathrm{keV}$. The resulting luminosity function and predicted distributions of flux and redshift were virtually identical to that from the simple power law with slope $\alpha_{23}$.

\section{Luminosity-Hardness Correlation}

Now that we have derived the GRB luminosity function, we are in a position to generate the luminosity-hardness correlation through a simulation. In order to reduce the effect of the counting errors on $\alpha_{23}$ discussed in Sec. 2.1, we use the $\alpha_{23}$ values of the brightest quartile, consisting of 348 GRBs with $<V / V_{\max }><0.069$. We assigned each of these GRBs a random luminosity from its spectral luminosity function $\Phi_{0}(L, s p)$. The resulting plot of peak luminosity versus $\alpha_{23}$ is shown in Figure 7. For $\alpha_{23}>-2.4$, we see clear evidence of the luminosity-hardness correlation, with a slope $d \log L / d \alpha_{23} \sim 1$.1. The dozen GRBs with a large range of spectral indices below $\alpha_{23}$ $<-2.4$ belong to the softest spectral class and therefore based on the present study cannot show any correlation between luminosity and hardness.

None of the GRBs observed by BATSE with redshifts listed by Lamb \& Reichart (2000) belong to the softest quartile in the BATSE catalog. Therefore, we cannot at the present time check the

main trend from soft to hard bursts in Figure 7. The total range in luminosity of a factor of 50 in the Lamb \& Reichart (2000) list is compatible with the range shown by the hard GRBs in Figure 7. This may indicate post facto that the adopted value of $\sigma_{\log L}=0.4$ is a reasonable one.

\section{Discussion}

We have shown that there exists for GRBs in the BD2 sample a $<V / V_{\max }>$-hardness correlation, see Figure 2. Since the BD2 sample was selected from BATSE DISCLA data on a timescale of $1024 \mathrm{~ms}$, the correlation applies to GRBs with durations larger than $1 \mathrm{~s}$. Based on the realization that the Euclidean value of $\left\langle V / V_{\max }\right\rangle$ is a cosmological distance indicator, we concluded that there exists a luminosity-hardness correlation, but could only demonstrate it after we had derived the luminosity function.

With the $<V / V_{\max }>$-hardness correlation we were able to derive the luminosity function without having to make assumptions about its overall shape, as had been required till now (Schmidt 1999b). We assumed a width for the luminosity function of each spectral class that was sufficient 
to produce a reasonably smooth overall luminosity function. The entire excercise of deriving the luminosity function was carried out without using any redshifts. We did have to make assumptions about the cosmological evolution or density distribution of GRBs for which we chose various star formation rate models.

This study has only been possible due to the power of the Euclidean value of $\left\langle V / V_{\max }\right\rangle$ as an independent cosmological distance indicator. Major progress beyond this work can be expected once systematic redshift surveys of GRBs become available. The luminosity-hardness correlation should become directly observable. From samples with observed redshifts complete to given flux limits the GRB luminosity function can be derived without having to make assumptions about the density distribution. And while the Euclidean $\left\langle V / V_{\max }\right\rangle$ cannot compete with the redshift as a distance indicator, the luminosity function should be compatible with the observed $<V / V_{\max }>$ values of the samples.

It is a pleasure to thank J. Brainerd, M. Finger and G. Pendleton for information about the calibration of the BATSE detectors, J. Trümper for useful discussions and A. Iyudin for raising questions about the statistical errors of the spectral index.

\section{REFERENCES}

Bahcall, N. A., Ostriker, J. P., Perlmutter, S., \& Steinhardt, P. J. 1999, Science, 284, 1481

Band, D. L., et al. 1993, ApJ, 413, 281

Belli, B. M. 1992, in AIP Conf. Proc. 265, Gamma-Ray Bursts, ed. W. Paciesas \& G. J. Fishman (New York: AIP), 100

Belli, B. M. 1996, in AIP Conf. Proc. 384, Gamma-Ray Bursts, ed. C. Kouveliotou, M. F. Briggs, \& G. J. Fishman (New York: AIP), 316

Belli, B. M. 2000, in AIP Conf. Proc. 526, Gamma-Ray Bursts, ed. R. M. Kippen, R. S. Mallozzi, \& G. J. Fishman (New York: AIP), 160

Blaine, A. W., Kneib, J-P., Ivison, R. J., \& Smail, I. 1999, ApJ, 512, L87

Cappellaro, E., et al. 1997, A\&A, 322, 431

Cohen, E., \& Piran, T. 1995, ApJ, 444, L25

Fenimore, E. E., \& Ramirez-Ruiz, E. 2000, ApJ, submitted, (astro-ph/0004176)

Fishman, G. J. et al. 1989, in GRO Science Workshop Proc., ed. W. N. Johnson (Greenbelt:NASA), $2-39$ 
Hakkila, J., Meegan, C. A., Pendleton, G. N., Malozzi, R. S., Haglin, D. J. \& Roiger, R. J. 2000, in AIP Conf. Proc. 526, Gamma-Ray Bursts, ed. R. M. Kippen, R. S. Mallozzi, \& G. J. Fishman (New York: AIP), 48

Kouveliotou, C., Meegan, C. A., Fishman, G. J., Bhat, N. P., Briggs, M. S., Koshut, T. M., Paciesas, W. S., \& Pendleton, G. N. 1993, ApJ, 413, L101

Kouveliotou, C., Koshut, T., Briggs, M. S., Pendleton, G. N., Meegan, C. A., Fishman, G. J., \& Lestrade, J. P. 1996, in AIP Conf. Proc. 384, Gamma-Ray Bursts, ed. C. Kouveliotou, M. F. Briggs, \& G. J. Fishman (New York: AIP), 42

Lamb, D. Q., \& Reichart, D. E. 2000, ApJ, 536, 1

Madau, P., \& Pozetti, L. 2000, MNRAS, 312, L9

Norris, J. P., Marani, G. F., \& Bonnell, J. T. 2000, ApJ, 534, 248

Pendleton, G. N., et al. 1996, ApJ, 464, 606

Pendleton, G. N., Evans, R. H., Mallozzi, R. S., Hakkila, J., Meegan, C. A., Paciesas, W. S., Preece, R. D., \& Briggs, M. S. 1998, in AIP Conf. Proc. 428, Gamma-Ray Bursts, ed. C. A. Meegan, R. D. Preece, \& T. M. Koshut (New York: AIP), 25

Porciani, C., \& Madau, P. 2000, submitted, (astro-ph/0008294)

Schmidt, M., 1968, ApJ, 151, 393

Schmidt, M., 1999a, A\&ASuppl, 138, 409

Schmidt, M., 1999b, ApJ, 523, L117

Steidel, C. C., et al. 1999, ApJ, 519, 1

Tavani, M. 1998, in AIP Conf. Proc. 428, Gamma-Ray Bursts, ed. C. A. Meegan, R. D. Preece, \& T. M. Koshut (New York: AIP), 72

Totani, T. 1999, ApJ, 511, 41

Wijers, R. A. M. J., Bloom, J. S., Bagla, J. S., \& Natarajan, P. 1998, MNRAS, 294, L13 
Table 1. $<V / V_{\max }>$ values as a function of $\left\langle\alpha_{23}>\right.$ in the BD2 sample.

\begin{tabular}{ccccc}
\hline \hline number & $<\alpha_{23}>_{\text {obs }}$ & $\left\langle V / V_{\max }>_{\text {obs }}\right.$ & $\left\langle\alpha_{23}>_{\text {corr }}\right.$ & $\left.<V / V_{\max }\right\rangle_{\text {corr }}$ \\
\hline 348 & -2.55 & $0.468 \pm 0.017$ & -2.33 & 0.421 \\
348 & -1.84 & $0.309 \pm 0.016$ & -1.79 & 0.325 \\
347 & -1.47 & $0.299 \pm 0.016$ & -1.47 & 0.344 \\
348 & -1.04 & $0.270 \pm 0.015$ & -1.10 & 0.256 \\
\hline
\end{tabular}




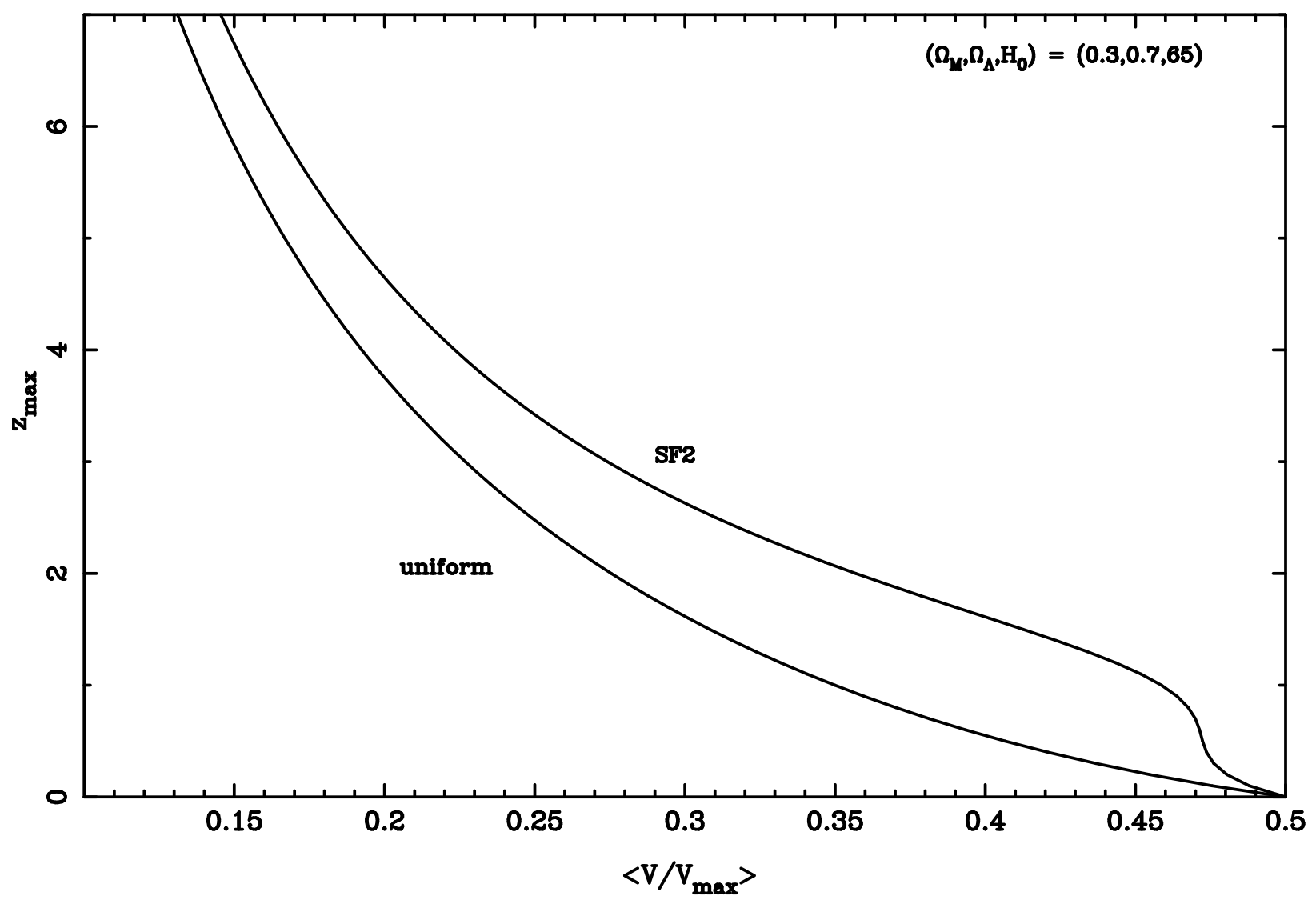

Fig. 1. - Illustration of the use of the Euclidean value of $\left\langle V / V_{\max }\right\rangle$ as a cosmological distance indicator. The objects used are standard candle bursts with a photon spectrum $E^{-2}$ in a cosmological model as indicated. The co-moving space density is taken to be uniform, or to be proportional to the star formation rate derived by Steidel et al. (1999) (model SF2, see Sec. 3). Plotted is the maximum redshift $z_{\max }$ of a sample of bursts versus the sample $<V / V_{\max }>$. 


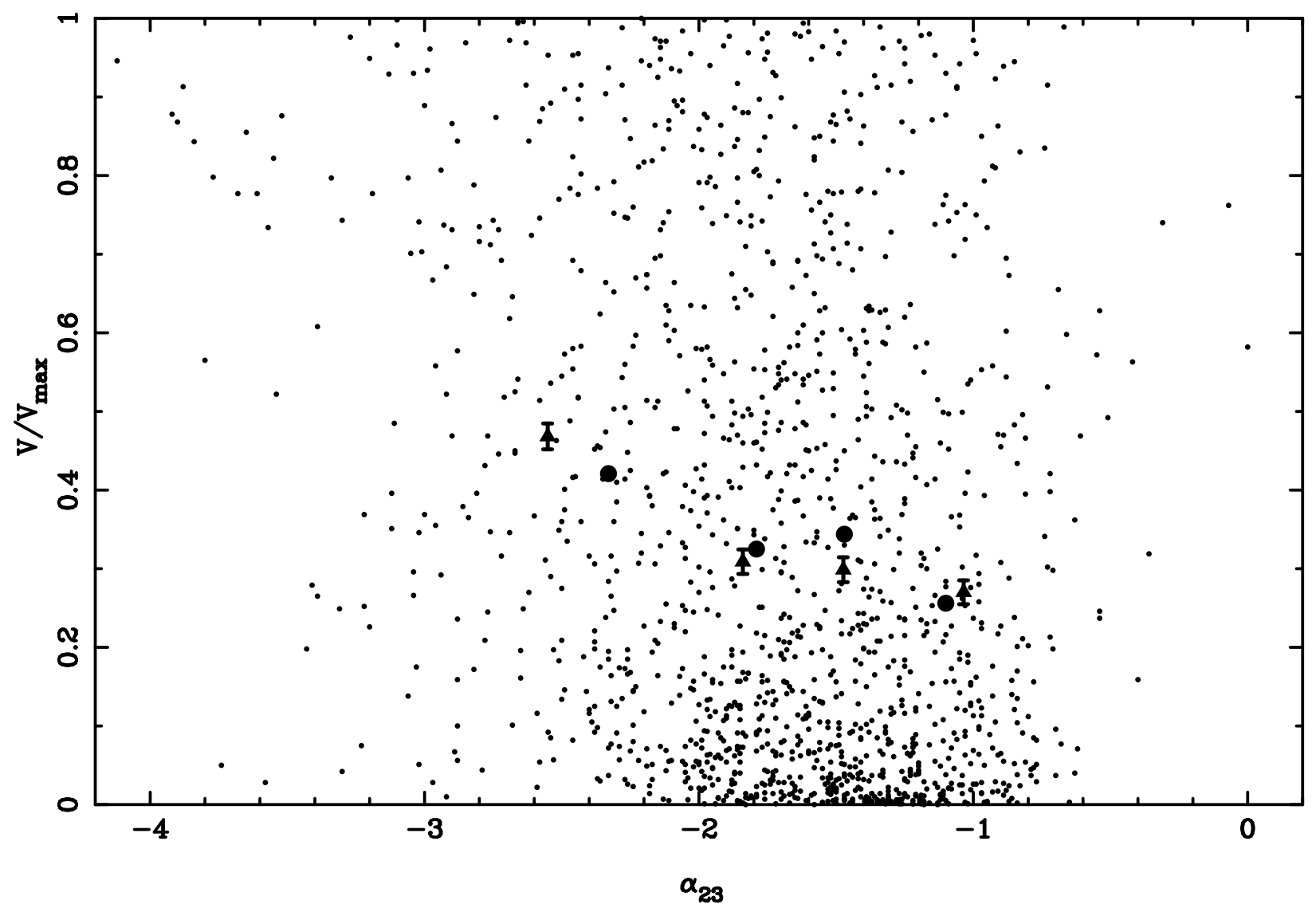

Fig. 2.- Plot of Euclidean values of $V / V_{\max }$ vs. spectral slope $\alpha_{23}$ for 1391 GRBs in the BD2 sample. The triangles show $<V / V_{\max }>$ for 4 spectral classes of $\sim 348$ objects each with error bars denoting the mean errors of the $<V / V_{\max }>$ values. The large dots show the mean values of $<\alpha_{23}>$ and $<V / V_{\max }>$ corrected for the effect of statistical errors in the peak counts. 


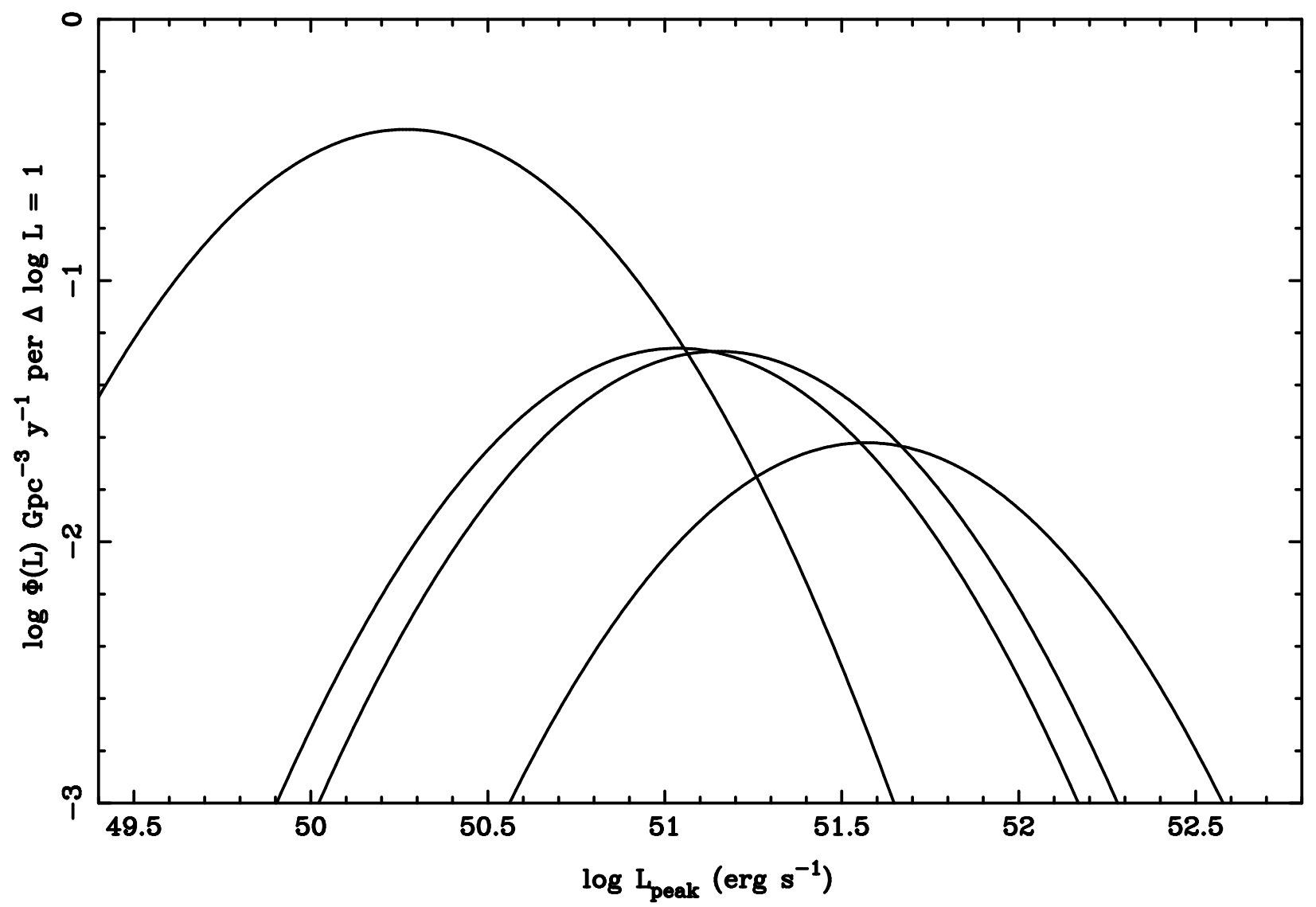

Fig. 3. - Luminosity functions at $z=0$ derived for 4 spectral classes for density distribution SF2. $L_{\text {peak }}$ is the peak luminosity in the energy range $50-300 \mathrm{keV}$. Each of the luminosity functions is a gaussian with $\sigma_{\log L}=0.4$. The central luminosities are derived from the Euclidean $<V / V_{\max }>$ values. 


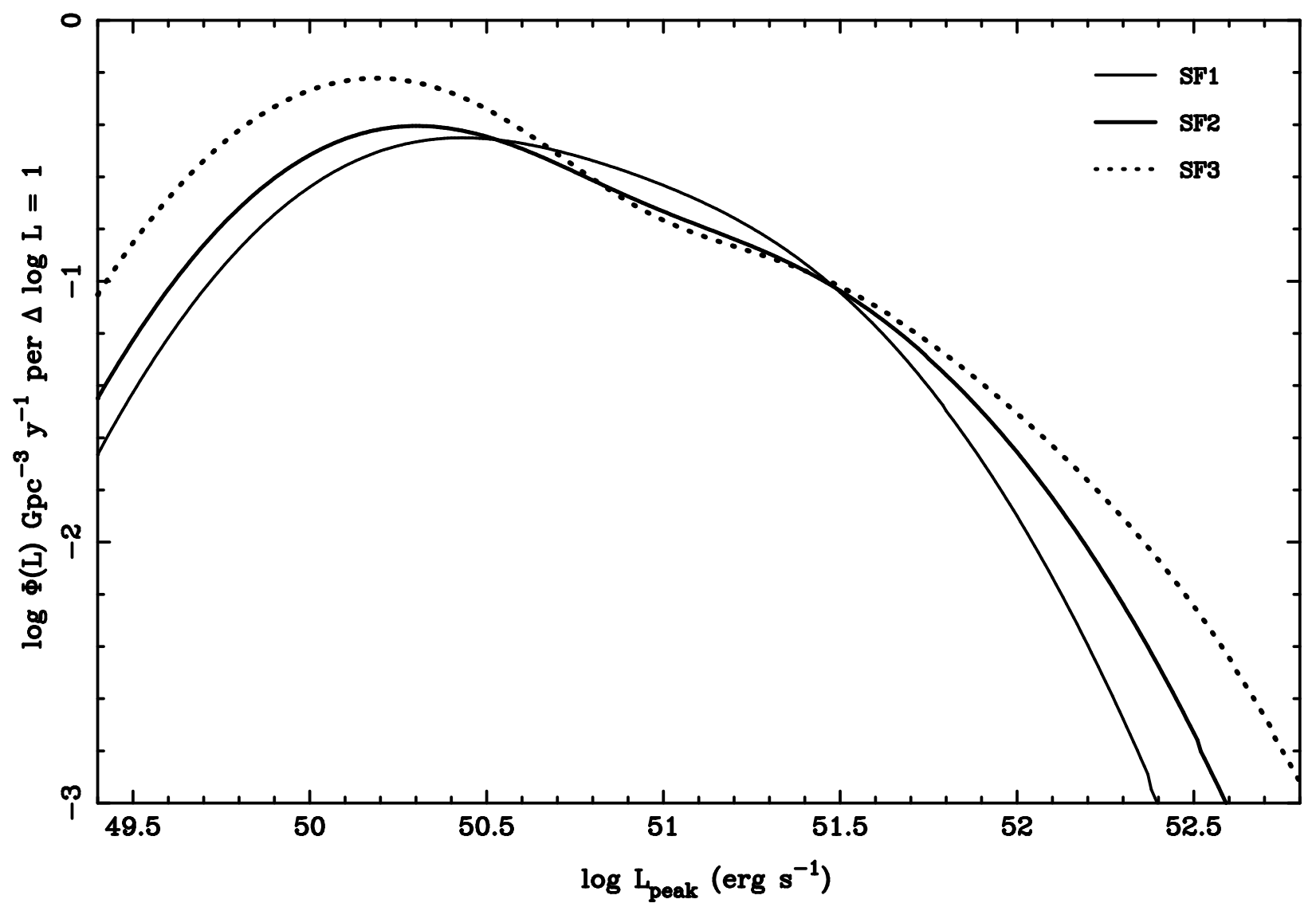

Fig. 4.- GRB luminosity functions at $z=0$ for density distributions SF1, SF2, and SF3, obtained as the sum of the 4 spectral luminosity functions. $L_{\text {peak }}$ is the peak luminosity in the energy range $50-300 \mathrm{keV}$. The luminosities and densities are 'isotropic-equivalent' values. 


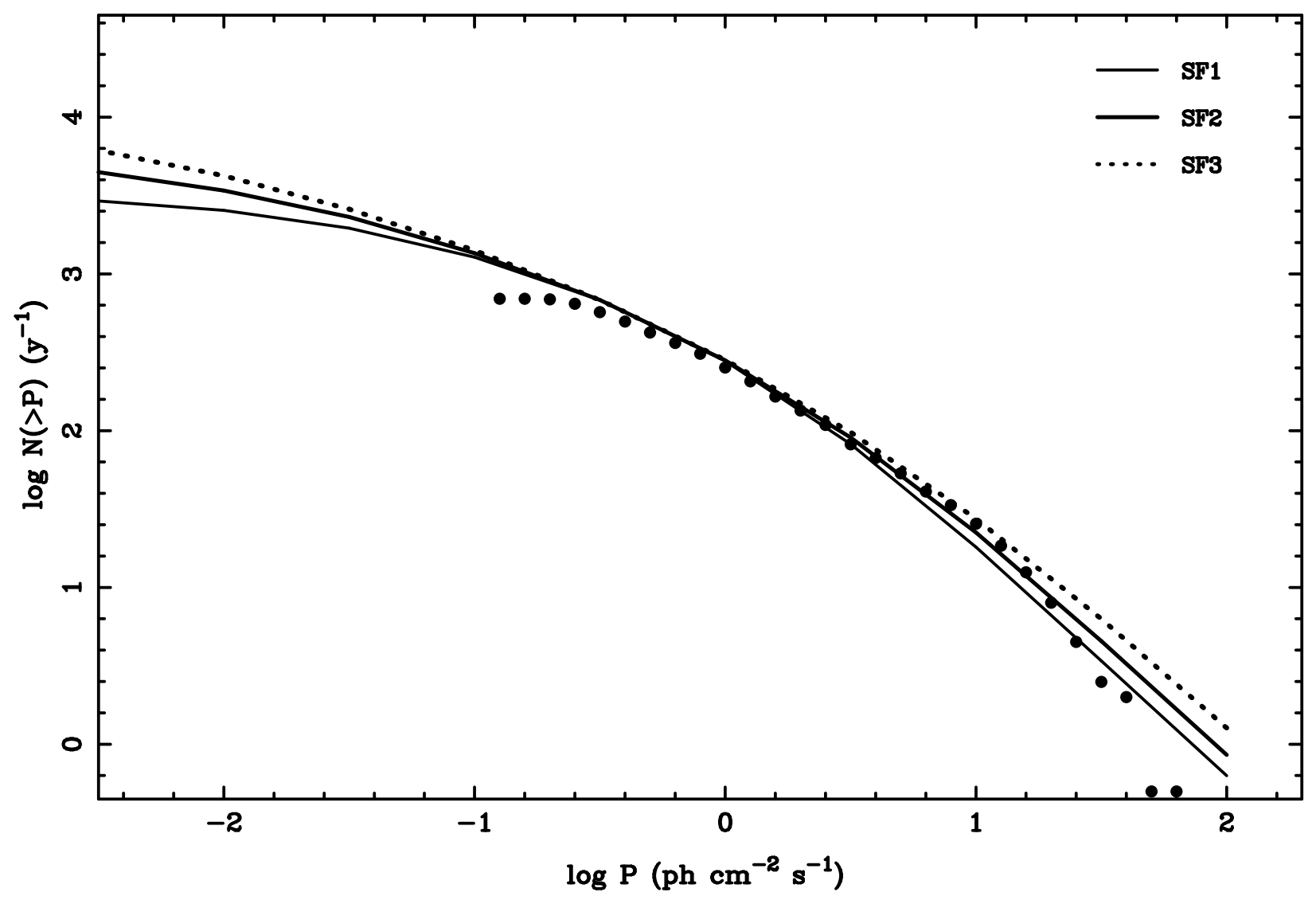

Fig. 5.- Predicted $\log N-\log P$ distribution for GRBs based on the luminosity functions shown in Figure 4. $P$ is the peak flux in the energy range $50-300 \mathrm{keV}$. The observed numbers in the BD2 sample are indicated as dots. 


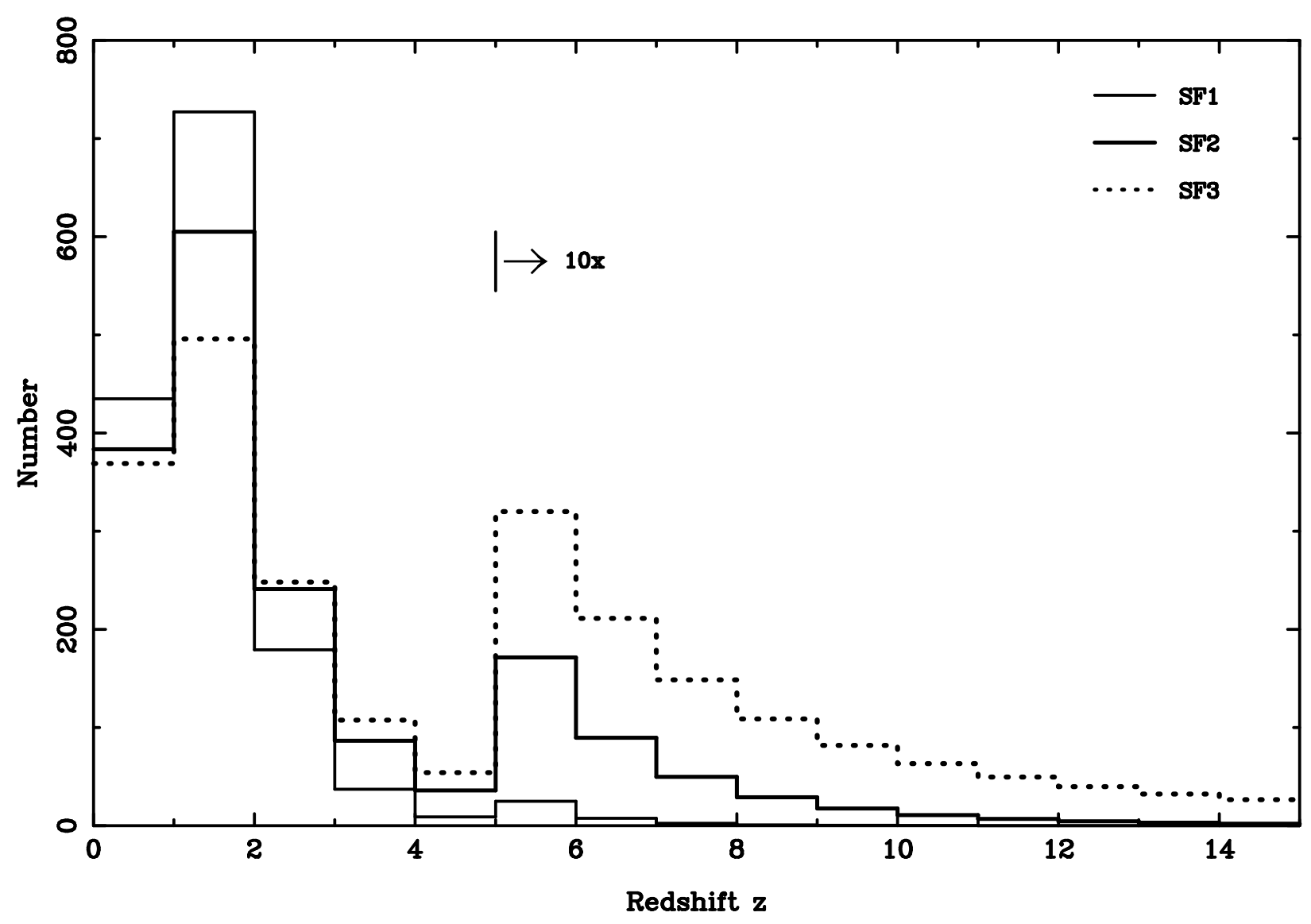

Fig. 6.- Predicted redshift distribution for the 1391 GRBs in the BD2 sample, based on the luminosity functions shown in Figure 4. Numbers plotted are multiplied by 10 for $z>5$. 


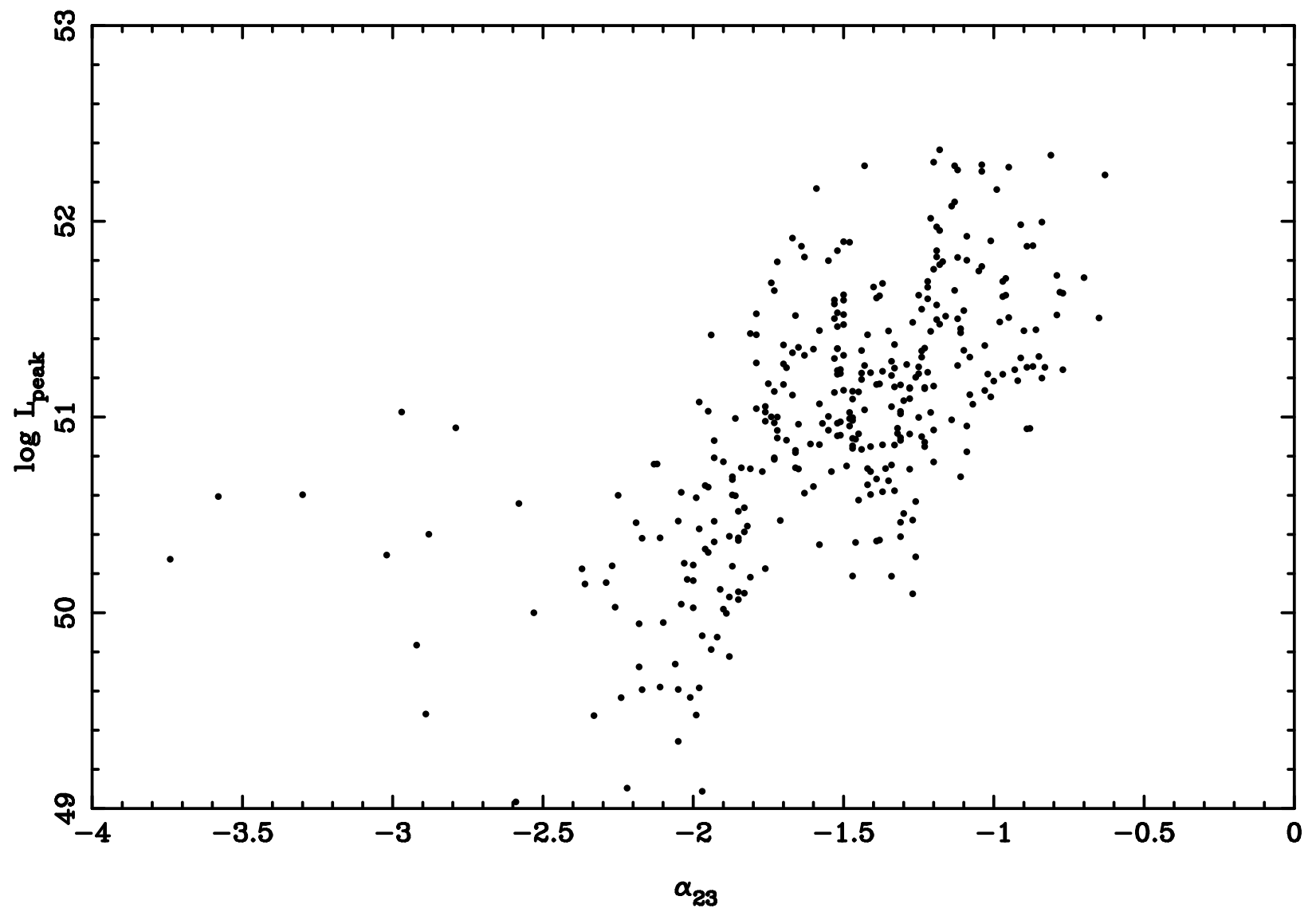

Fig. 7.- Luminosity-hardness correlation of GRBs, produced by a simulation using the luminosity function based on the SF2 density distribution (see Figs. 3 and 4) and $\sigma_{\log L}=0.4$. 